\title{
Awards at the International Congress of Mathematicians at Seoul (Korea) 2014
}

\section{News release from the International Mathematical Union (IMU)}

The Fields medals of the ICM 2014 were awarded to Arthur Avila, Manjual Bhagava, Martin Hairer, and Maryam Mirzakhani; and the Nevanlinna Prize was awarded to Subhash Khot. The prize winners of the Gauss Prize, Chern Prize, and Leelavati Prize were Stanley Osher, Phillip Griffiths and Adrián Paenza, respectively. The following article about the awardees is the news released by the International Mathematical Union (IMU). We are grateful to Prof. Ingrid Daubechies, the President of IMU, who granted us the permission of reprinting it.-the Editors

\section{The Work of Artur Avila}

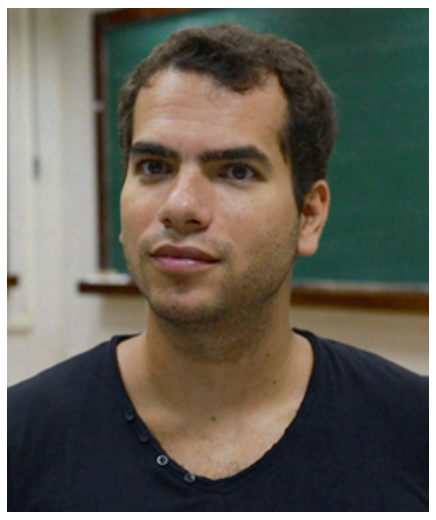

Artur Avila has made outstanding contributions to dynamical systems, analysis, and other areas, in many cases proving decisive results that solved long-standing open problems. A native of Brazil who spends part of his time there and part in France, he combines the strong mathematical cultures and traditions of both countries. Nearly all his work has been done through collaborations with some 30 mathematicians around the world. To these collaborations Avila brings formidable technical power, the ingenuity and tenacity of a master problem-solver, and an unerring sense for deep and significant questions.

Avila's achievements are many and span a broad range of topics; here we focus on only a few highlights. One of his early significant results closes a chapter on a long story that started in the 1970s. At that time, physicists, most notably Mitchell Feigenbaum, began trying to understand how chaos can arise out of very simple systems. Some of the systems they looked at were based on iterating a mathematical rule such as $3 x(1-x)$. Starting with a given point, one can watch the trajectory of the point under repeated applications of the rule; one can think of the rule as moving the starting point around over time. For some maps, the trajectories eventually settle into stable orbits, while for other maps the trajectories become chaotic.

Out of the drive to understand such phenomena grew the subject of discrete dynamical systems, to which scores of mathematicians contributed in the ensuing decades. Among the central aims was to develop ways to predict long-time behavior. For a trajectory that settles into a stable orbit, predicting where a point will travel is straight-forward. But not for a chaotic trajectory: Trying to predict exactly where an initial point goes after a long time is akin to trying to predict, after a million tosses of a coin, whether the million-and-first toss will be a head or a tail. But one can model coin-tossing probabilistically, using stochastic tools, and one can try to do the same for trajectories. Mathematicians noticed that many 
of the maps that they studied fell into one of two categories: "regular", meaning that the trajectories eventually become stable, or "stochastic", meaning that the trajectories exhibit chaotic behavior that can be modeled stochastically. This dichotomy of regular vs. stochastic was proved in many special cases, and the hope was that eventually a more-complete understanding would emerge. This hope was realized in a 2003 paper by Avila, Welington de Melo, and Mikhail Lyubich, which brought to a close this long line of research. Avila and his co-authors considered a wide class of dynamical systems-namely, those arising from maps with a parabolic shape, known as unimodal maps-and proved that, if one chooses such a map at random, the map will be either regular or stochastic. Their work provides a unified, comprehensive picture of the behavior of these systems.

Another outstanding result of Avila is his work, with Giovanni Forni, on weak mixing. If one attempts to shuffle a deck of cards by only cutting the deck-that is, taking a small stack off the top of the deck and putting the stack on the bottom-then the deck will not be truly mixed. The cards are simply moved around in a cyclic pattern. But if one shuffles the cards in the usual way, by interleaving them-so that, for example, the first card now comes after the third card, the second card after the fifth, and so on-then the deck will be truly mixed. This is the essential idea of the abstract notion of mixing that Avila and Forni considered. The system they worked with was not a deck of cards, but rather a closed interval that is cut into several subintervals. For example, the interval could be cut into four pieces, $\mathrm{ABCD}$, and then one defines a map on the interval by exchanging the positions of the subintervals so that, say, ABCD goes to DCBA. By iterating the map, one obtains a dynamical system called an "interval exchange transformation".

Considering the parallel with cutting or shuffling a deck of cards, one can ask whether an interval exchange transformation can truly mix the subintervals. It has long been known that this is impossible. However, there are ways of quantifying the degree of mixing that lead to the notion of "weak mixing", which describes a system that just barely fails to be truly mixing. What Avila and Forni showed is that almost every interval exchange transformation is weakly mixing; in other words, if one chooses at random an interval exchange transformation, the overwhelmingly likelihood is that, when iterated, it will produce a dynamical system that is weakly mixing. This work is connected to more-recent work by Avila and Vincent Delecroix, which investigates mixing in regular polygonal billiard systems. Billiard systems are used in statistical physics as models of particle motion. Avila and Delecroix found that almost all dy- namical systems arising in this context are weakly mixing.

In the two lines of work mentioned above, Avila brought his deep knowledge of the area of analysis to bear on questions in dynamical systems. He has also sometimes done the reverse, applying dynamical systems approaches to questions in analysis. One example is his work on quasi-periodic Schrödinger operators. These are mathematical equations for modeling quantum mechanical systems. One of the emblematic pictures from this area is the Hofstadter butterfly, a fractal pattern named after Douglas Hofstadter, who first came upon it in 1976. The Hofstadter butterfly represents the energy spectrum of an electron moving under an extreme magnetic field.

Physicists were stunned when they noticed that, for certain parameter values in the Schrödinger equation, this energy spectrum appeared to be the Cantor set, which is a remarkable mathematical object that embodies seemingly incompatible properties of density and sparsity. In the 1980s, mathematician Barry Simon popularized the "Ten Martini Problem" (so named by Mark Kac, who offered to buy 10 martinis for anyone who could solve it). This problem asked whether the spectrum of one specific Schrödinger operator, known as the almost-Mathieu operator, is in fact the Cantor set. Together with Svetlana Jitomirskaya, Avila solved this problem.

As spectacular as that solution was, it represents only the tip of the iceberg of Avila's work on Schrödinger operators. Starting in 2004, he spent many years developing a general theory that culminated in two preprints in 2009. This work establishes that, unlike the special case of the almost-Mathieu operator, general Schrödinger operators do not exhibit critical behavior in the transition between different potential regimes. Avila used approaches from dynamical systems theory in this work, including renormalization techniques.

A final example of Avila's work is a very recent result that grew out of his proof of a regularization theorem for volume-preserving maps. This proof resolved a conjecture that had been open for thirty years; mathematicians hoped that the conjecture was true but could not prove it. Avila's proof has unblocked a whole direction of research in smooth dynamical systems and has already borne fruit. In particular, the regularization theorem is a key element in an important recent advance by Avila, Sylvain Crovisier, and Amie Wilkinson. Their work, which is still in preparation, shows that a generic volumepreserving diffeomorphism with positive metric entropy is an ergodic dynamical system.

With his signature combination of tremendous analytical power and deep intuition about dynamical systems, Artur Avila will surely remain a mathematical leader for many years to come. 


\section{References}

A. Avila, W. de Melo, and M. Lyubich, "Regular or stochastic dynamics in real analytic families of unimodal maps", Inventiones Mathematicae, 2003.

A. Avila and G. Forni, "Weak mixing for interval exchange transformations and translation flows", Annals of Mathematics, 2007.

A. Avila and V. Delecroix, "Weak mixing directions in non-arithmetic Veech surfaces”, preprint 2013.

A. Avila and S. Jitomirskaya, "The Ten Martini Problem”, Annals of Mathematics, 2009.

A. Avlia, "Global theory of one-frequency Schrödinger operators I, II”, preprints 2009.

A. Avila, "On the regularization of conservative maps", Acta Mathematica, 2010.

\section{Biography}

Born in Brazil in 1979, Artur Avila is also a naturalized French citizen. He received his $\mathrm{PhD}$ in 2001 from the Instituto Nacional de Matemática Pura e Aplicada (IMPA) in Rio de Janeiro, where his advisor was Welington de Melo. Since 2003 Avila has been researcher in the Centre National de la Recherche Scientifique and became a Directeur de recherche in 2008; he is attached to the Institut de Mathématiques de Jussieu-Paris Rive Gauche. Also, since 2009 he has been a researcher at IMPA. Among his previous honors are the Salem Prize (2006), the European Mathematical Society Prize (2008), the Grand Prix Jacques Herbrand of the French Academy of Sciences (2009), the Michael Brin Prize (2011), the Prêmio of the Sociedade Brasileira de Matemática (2013), and the TWAS Prize in Mathematics (2013) of the World Academy of Sciences.

\section{The Work of Manjul Bhargava}

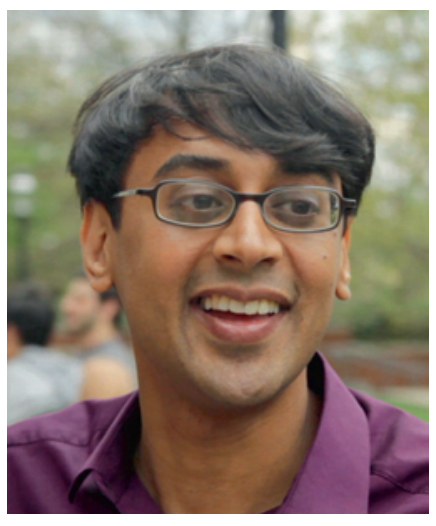

Manjul Bhargava's work in number theory has had a profound influence on the field. A mathematician of extraordinary creativity, he has a taste for simple problems of timeless beauty, which he has solved by developing elegant and powerful new methods that offer deep

insights.

When he was a graduate student, Bhargava read the monumental Disquisitiones Arithmeticae, a book about number theory by Carl Friedrich Gauss (1777-1855). All mathematicians know of the Disquisitiones, but few have actually read it, as its notation and computational nature make it difficult for modern readers to follow. Bhargava nevertheless found the book to be a wellspring of inspiration. Gauss was interested in binary quadratic forms, which are polynomials $a x^{2}+b x y+c y^{2}$, where $a, b$, and $c$ are integers. In the Disquisitiones, Gauss developed his ingenious composition law, which gives a method for composing two binary quadratic forms to obtain a third one. This law became, and remains, a central tool in algebraic number theory. After wading through the 20 pages of Gauss's calculations culminating in the composition law, Bhargava knew there had to be a better way.

Then one day, while playing with a Rubik's cube, he found it. Bhargava thought about labeling each corner of a cube with a number and then slicing the cube to obtain 2 sets of 4 numbers. Each 4 -number set naturally forms a matrix. A simple calculation with these matrices resulted in a binary quadratic form. From the three ways of slicing the cube, three binary quadratic forms emerged. Bhargava then calculated the discriminants of these three forms. (The discriminant, familiar to some as the expression "under the square root sign" in the quadratic formula, is a fundamental quantity associated to a polynomial.) When he found the discriminants were all the same, as they are in Gauss's composition law, Bhargava realized he had found a simple, visual way to obtain the law.

He also realized that he could expand his cubelabeling technique to other polynomials of higher degree (the degree is the highest power appearing in the polynomial; for example, $x^{3}-x+1$ has degree 3 ). He then discovered 13 new composition laws for higherdegree polynomials. Up until this time, mathematicians had looked upon Gauss's composition law as a curiosity that happened only with binary quadratic forms. Until Bhargava's work, no one realized that other composition laws existed for polynomials of higher degree.

One of the reasons Gauss's composition law is so important is that it provides information about quadratic number fields. A number field is built by extending the rational numbers to include non-rational roots of a polynomial; if the polynomial is quadratic, then one obtains a quadratic number field. The degree of the polynomial and its discriminant are two basic quantities associated with the number field. Although number fields are fundamental objects in algebraic number theory, some basic facts are unknown, such as how many number fields there are for a fixed degree and fixed discriminant. With his new composition laws in hand, Bhargava set about using them to investigate number fields.

Implicit in Gauss's work is a technique called the "geometry of numbers"; the technique was more fully developed in a landmark 1896 work of Hermann Minkowski (1864-1909). In the geometry of numbers, one imagines the plane, or 3-dimensional space, as populated by a lattice that highlights points with in- 
teger coordinates. If one has a quadratic polynomial, counting the number of integer lattice points in a certain region of 3-dimensional space provides information about the associated quadratic number field. In particular, one can use the geometry of numbers to show that, for discriminant with absolute value less than $X$, there are approximately $X$ quadratic number fields. In the 1960s, a more refined geometry of numbers approach by Harold Davenport (1907-1969) and Hans Heilbronn (1908-1975) resolved the case of degree 3 number fields. And then progress stopped. So a great deal of excitement greeted Bhargava's work in which he counted the number of degree 4 and degree 5 number fields having bounded discriminant. These results use his new composition laws, together with his systematic development of the geometry of numbers, which greatly extended the reach and power of this technique. The cases of degree bigger than 5 remain open, and Bhargava's composition laws will not resolve those. However, it is possible that those cases could be attacked using analogues of his composition laws.

Recently, Bhargava and his collaborators have used his expansion of the geometry of numbers to produce striking results about hyperelliptic curves. At the heart of this area of research is the ancient question of when an arithmetic calculation yields a square number. One answer Bhargava found is strikingly simple to state: A typical polynomial of degree at least 5 with rational coefficients never takes a square value. A hyperelliptic curve is the graph of an equation of the form $y^{2}=$ a polynomial with rational coefficients. In the case where the polynomial has degree 3, the graph is called an elliptic curve. Elliptic curves have especially appealing properties and have been the subject of a great deal of research; they also played a prominent role in Andrew Wiles's celebrated proof of Fermat's Last Theorem.

A key question about a hyperelliptic curve is how one can count the number of points that have rational coordinates and that lie on the curve. It turns out that the number of rational points is closely related to the degree of the curve. For curves of degree 1 and 2 , there is an effective way of finding all the rational points. For degree 5 and higher, a theorem of Gerd Faltings (a 1986 Fields Medalist) says that there are only finitely many rational points. The most mysterious cases are those of degree 3-namely, the case of elliptic curves-and of degree 4. There is not even any algorithm known for deciding whether a given curve of degree 3 or 4 has finitely many or infinitely many rational points.

Such algorithms seem out of reach. Bhargava took a different tack and asked, what can be said about the rational points on a typical curve? In joint work with Arul Shankar and also with Christopher Skin- ner, Bhargava came to the surprising conclusion that a positive proportion of elliptic curves have only one rational point and a positive proportion have infinitely many. Analogously, in the case of hyperelliptic curves of degree 4, Bhargava showed that a positive proportion of such curves have no rational points and a positive proportion have infinitely many rational points. These works necessitated counting lattice points in unbounded regions of high-dimensional space, in which the regions spiral outward in complicated "tentacles". This counting could not have been done without Bhargava's expansion of the geometry of numbers technique.

Bhargava also used his expansion of the geometry of numbers to look at the more general case of higher degree hyperelliptic curves. As noted above, Faltings' theorem tells us that for curves of degree 5 or higher, the number of rational points is finite, but the theorem does not give any way of finding the rational points or saying exactly how many there are. Once again, Bhargava examined the question of what happens for a "typical" curve. When the degree is even, he found that the typical hyperelliptic curve has no rational points at all. Joint work with Benedict Gross, together with follow-up work of Bjorn Poonen and Michael Stoll, established the same result for the case of odd degree. These works also offer quite precise estimates of how quickly the number of curves having rational points decreases as the degree increases. For example, Bhargava's work shows that, for a typical degree 10 polynomial, there is a greater than $99 \%$ chance that the curve has no rational points.

A final example of Bhargava's achievements is his work with Jonathan Hanke on the so-called "290-Theorem". This theorem concerns a question that goes back to the time of Pierre de Fermat (1601-1665), namely, which quadratic forms represent all integers? For example, not all integers are the sum of two squares, so $x^{2}+y^{2}$ does not represent all integers. Neither does the sum of three squares, $x^{2}+y^{2}+z^{2}$. But, as Joseph-Louis Lagrange (1736-1813) famously established, the sum of four squares, $x^{2}+y^{2}+z^{2}+w^{2}$, does represent all integers. In 1916, Srinivasa Ramanujan (1887-1920) gave 54 more examples of such forms in 4 variables that represent all integers. What other such "universal" forms could be out there? In the early 1990s, John H. Conway and his students, particularly William Schneeberger and Christopher Simons, looked at this question a different way, asking whether there is a number $c$ such that, if a quadratic form represents integers less than $c$, it represents all integers. Through extensive computations, they conjectured that $c$ could perhaps be taken as small as 290 . They made remarkable progress, but it was not until Bhargava and Hanke took up the question that it was fully resolved. They 
found a set of 29 integers, up to and including 290, such that, if a quadratic form (in any number of variables) represents these 29 integers, then it represents all integers. The proof is a feat of ingenuity combined with extensive computer programming.

In addition to being one of the world's leading mathematicians, Bhargava is an accomplished musician; he plays the Indian instrument known as the tabla at a professional level. An outstanding communicator, he has won several teaching awards, and his lucid and elegant writing has garnered a prize for exposition.

Bhargava has a keen intuition that leads him unerringly to deep and beautiful mathematical questions. With his immense insight and great technical mastery, he seems to bring a "Midas touch" to everything he works on. He surely will bring more delights and surprises to mathematics in the years to come.

\section{References}

M. Bhargava, "Higher composition laws," parts I, II, and III, Annals of Mathematics, 2004; part IV, Annals of Mathematics, 2008.

M. Bhargava, "The density of discriminants of quartic rings and fields," Annals of Mathematics, 2005.

M. Bhargava, "The density of discriminants of quintic rings and fields," Annals of Mathematics, 2010.

M. Bhargava and B. Gross, "The average size of the 2-Selmer group of the Jacobians of hyperelliptic curves with a rational Weierstrass point", in: Automorphic Representations and L-functions, TIFR Studies in Mathematics, 2013.

M. Bhargava and A. Shankar, "Binary quartic forms having bounded invariants, and the boundedness of the average rank of elliptic curves," Annals of Mathematics, to appear.

M. Bhargava and A. Shankar, "Ternary cubic forms having bounded invariants and the existence of a positive proportion of elliptic curves having rank 0," Annals of Mathematics, to appear.

M. Bhargava and J. Hanke, "Universal quadratic forms and the 290-theorem", preprint 2011.

M. Bhargava, "Most hyperelliptic curves over Q have no rational points," preprint 2013, http://arxiv.org/abs/ 1308.0395.

B. Poonen and M. Stoll, "Most odd degree hyperelliptic curves have only one rational point," preprint 2013, http://arxiv.org/abs/1302.0061.

M. Bhargava, "A positive proportion of plane cubics fail the Hasse principle," http://arxiv.org/abs/1402. 1131, preprint 2014.

M. Bhargava and C. Skinner, "A positive proportion of elliptic curves over Q have rank one," preprint 2014, http:// arxiv.org/abs/1401.0233.

\section{Biography}

Born in 1974 in Canada, Manjul Bhargava grew up primarily in the USA and also spent much time in India. He received his $\mathrm{PhD}$ in 2001 from Princeton University, under the direction of Andrew Wiles. Bhargava became a professor at Princeton in 2003. His honors include the Merten M. Hasse Prize of the
Mathematical Association of America (2003), the Blumenthal Award for the Advancement of Research in Pure Mathematics (2005), the SASTRA Ramanujan Prize (2005), the Cole Prize in Number Theory of the American Mathematical Society (2008), the Fermat Prize (2011), and the Infosys Prize (2012). He was elected to the U.S. National Academy of Sciences in 2013.

\section{The Work of Martin Hairer}

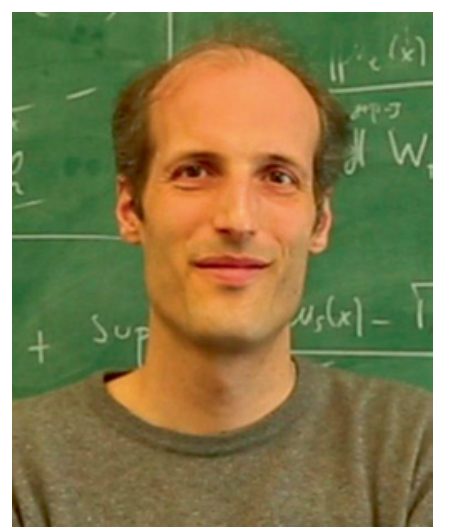

Martin Hairer has made a major breakthrough in the study of stochastic partial differential equations by creating a new theory that provides tools for attacking problems that up to now had seemed impenetrable.

The subject of differential equations has its roots in the development of calculus by Isaac Newton and Gottfried Leibniz in the 17 th century. A major motivation at that time was to understand the motion of the planets in the solar system. Newton's laws of motion can be used to formulate a differential equation that describes, for example, the motion of the Earth around the Sun. A solution to such an equation is a function that gives the position of the Earth at any time $t$. In the centuries since, differential equations have become ubiquitous across all areas of science and engineering to describe systems that change over time.

A differential equation describing planetary motion is deterministic, meaning that it determines exactly where a planet will be at a given time in the future. Other differential equations are stochastic, meaning that they describe systems containing an inherent element of randomness. An example is an equation that describes how a stock price will change over time. Such an equation incorporates a term that represents fluctuations in the stock market price. If one could predict exactly what the fluctuations would be, one could predict the future stock price exactly (and get very rich!). However, the fluctuations, while having some dependence on the initial stock price, are essentially random and unpredictable. The stockprice equation is an example of a stochastic differential equation.

In the planetary-motion equation, the system changes with respect to only one variable, namely, time. Such an equation is called an ordinary differential equation (ODE). By contrast, partial differential equations (PDEs) describe systems that change with respect to more than one variable, for example, time 
and position. Many PDEs are nonlinear, meaning that the terms in it are not simple proportions-for example, they might be raised to an exponential power. Some of the most important natural phenomena are governed by nonlinear PDEs, so understanding these equations is a major goal for mathematics and the sciences. However, nonlinear PDEs are among the most difficult mathematical objects to understand. Hairer's work has caused a great deal of excitement because it develops a general theory that can be applied to a large class of nonlinear stochastic PDEs.

An example of a nonlinear stochastic PDE-and one that played an important role in Hairer's work-is the KPZ equation, which is named for Mehran Kardar, Giorgio Parisi, and Yi-Cheng Zhang, the physicists who proposed the equation in 1986 for the motion of growing interfaces. To gain some insight into the nature of the equation, consider the following simplified model for ballistic deposition. Particles move towards a substrate and stick upon arrival; as a consequence, the substrate height grows linearly in time, at the same time becoming increasingly more rough. In this context the KPZ equation describes the time evolution of the interface between vacuum and accumulated material. The randomness in the arrival positions and times of the particles introduces a spacetime white noise into the equation, thus turning KPZ into a stochastic PDE, which describes the evolution over time of the rough, irregular interface between the vacuum above and the material accumulating below. A solution to the KPZ equation would provide, for any time $t$ and any point along the bottom edge of the substrate, the height of the interface above that point.

The challenge the KPZ equation posed is that, although it made sense from the point of view of physics, it did not make sense mathematically. A solution to the KPZ equation should be a mathematical object that represents the rough, irregular nature of the interface. Such an object has no smoothness; in mathematical terms, it is not differentiable. And yet two of the terms in the KPZ equation call for the object to be differentiable. There is a way to sidestep this difficulty by using an object called a distribution. But then a new problem arises, because the KPZ equation is nonlinear: It contains a square term, and distributions cannot be squared. For these reasons, the KPZ equation was not well defined. Although researchers came up with some technical tricks to ameliorate these difficulties for the special case of the KPZ equation, the fundamental problem of its not being well defined long remained an unresolved issue.

In a spectacular achievement, Hairer overcame these difficulties by describing a new approach to the KPZ equation that allows one to give a mathematically precise meaning to the equation and its solutions.
What is more, in subsequent work he used the ideas he developed for the KPZ equation to build a general theory, the theory of regularity structures, that can be applied to a broad class of stochastic PDEs. In particular, Hairer's theory can be used in higher dimensions.

The basic idea of Hairer's approach to the KPZ equation is the following. Instead of making the usual assumption that the small random effects occur on an infinitesimally small scale, he adopted the assumption that the random effects occur on a scale that is small in comparison to the scale at which the system is viewed. Removing the infinitesimal assumption, which Hairer calls "regularizing the noise", renders an equation that can be solved. The resulting solution is not a solution to KPZ; rather, it can be used as the starting point to construct a sequence of objects that, in the limit, converges to a solution of KPZ. And Hairer proved a crucial fact: the limiting solution is always the same regardless of the kind of noise regularization that is used.

Hairer's general theory addresses other, higherdimensional stochastic PDEs that are not well defined. For these equations, as with KPZ, the main challenge is that, at very small scales, the behavior of the solutions is very rough and irregular. If the solution were a smooth function, one could carry out a Taylor expansion, which is a way of approximating the function by polynomials of increasingly higher degree. But the roughness of the solutions means they are not well approximated by polynomials. What Hairer did instead is to define objects, custom-built for the equation at hand, that approximate the behavior of the solution at small scales. These objects then play a role similar to polynomials in a Taylor expansion. At each point, the solution will look like an infinite superposition of these objects. The ultimate solution is then obtained by gluing together the pointwise superpositions. Hairer established the crucial fact that the ultimate solution does not depend on the approximating objects used to obtain it.

Prior to Hairer's work, researchers had made a good deal of progress in understanding linear stochastic PDEs, but there was a fundamental block to addressing nonlinear cases. Hairer's new theory goes a long way towards removing that block. What is more, the class of equations to which the theory applies contains several that are of central interest in mathematics and science. In addition, his work could open the way to understanding the phenomenon of universality. Other equations, when rescaled, converge to the KPZ equation, so there seems to be some universal phenomenon lurking in the background. Hairer's work has the potential to provide rigorous analytical tools to study this universality.

Before developing the theory of regularity structures, Hairer made other outstanding contributions. 
For example, his joint work with Jonathan Mattingly constitutes a significant advance in understanding a stochastic version of the Navier-Stokes equation, a nonlinear PDE that describes wave motion. In addition to being one of the world's top mathematicians, Hairer is a very good computer programmer. While still a school student, he created audio editing software that he later developed and successfully marketed as "the Swiss army knife of sound editing". His mathematical work does not depend on computers, but he does find that programming small simulations helps develop intuition.

With his commanding technical mastery and deep intuition about physical systems, Hairer is a leader in the field who will doubtless make many further significant contributions.

\section{References}

M. Hairer, "Solving the KPZ equation", Annals of Mathematics, 2013.

M. Hairer, "A theory of regularity structures", Inventiones Mathematicae, 2014.

\section{Biography}

Born in 1975, Martin Hairer is an Austrian citizen. In 2001, he received his $\mathrm{PhD}$ in physics from the University of Geneva, under the direction of Jean-Pierre Eckmann. He is currently Regius Professor of Mathematics at the University of Warwick. His honors include the Whitehead Prize of the London Mathematical Society (2008), the Philip Leverhulme Prize (2008), the Wolfson Research Merit Award of the Royal Society (2009), the Fermat Prize (2013), and the Fröhlich Prize of the London Mathematical Society (2014). He was elected a Fellow of the Royal Society in 2014.

\section{The Work of Maryam Mirzakhani}

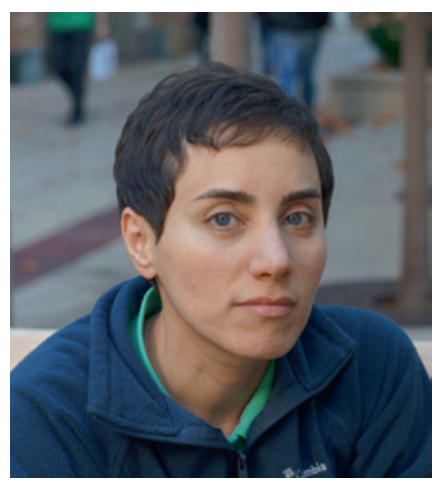

Maryam Mirzakhani has made striking and highly original contributions to geometry and dynamical systems. Her work on Riemann surfaces and their moduli spaces bridges several mathematical disciplineshyperbolic geometry, complex analysis, to-

pology, and dynamics-and influences them all in return. She gained widespread recognition for her early results in hyperbolic geometry, and her most recent work constitutes a major advance in dynamical systems.
Riemann surfaces are named after the 19th century mathematician Bernhard Riemann, who was the first to understand the importance of abstract surfaces, as opposed to surfaces arising concretely in some ambient space. Mathematicians building on Riemann's insights understood more than 100 years ago that such surfaces can be classified topologically, i.e. up to deformation, by a single number, namely, the number of handles. This number is called the genus of the surface. The sphere has genus zero, the surface of a coffee cup has genus one, and the surface of a proper pretzel has genus three. Provided that one disregards the precise geometric shape, there is exactly one surface of genus $g$ for every positive integer $g$.

A surface becomes a Riemann surface when it is endowed with an additional geometric structure. One can think of this geometric structure as a socalled complex structure, which allows one to do complex analysis on the abstract surface. Since the complex numbers involve two real parameters, a surface, which is two-dimensional over the real numbers, has only one complex dimension and is sometimes called a complex curve. The following fact links the theory of Riemann surfaces to algebraic geometry: Every complex curve is an algebraic curve, meaning that the complex curve, although defined abstractly, can be realized as a curve in a standard ambient space, in which it is the zero set of suitably chosen polynomials. Thus, although a Riemann surface is a priori an analytic object defined in terms of complex analysis on abstract surfaces, it turns out to have an algebraic description in terms of polynomial equations.

An alternative but equivalent way of defining a Riemann surface is through the introduction of a geometry that allows one to measure angles, lengths, and areas. The most important such geometry is hyperbolic geometry, the original example of a nonEuclidean geometry discovered by Bolyai, Gauss, and Lobatchevski. The equivalence between complex algebraic and hyperbolic structures on surfaces is at the root of the rich theory of Riemann surfaces.

Mirzakhani's early work concerns closed geodesics on a hyperbolic surface. These are closed curves whose length cannot be shortened by deforming them. A now-classic theorem proved more than 50 years ago gives a precise way of estimating the number of closed geodesics whose length is less than some bound $L$. The number of closed geodesics grows exponentially with $L$; specifically, it is asymptotic to $e^{L} / L$ for large $L$. This theorem is called the "prime number theorem for geodesics", because it is exactly analogous to the usual "prime number theorem" for whole numbers, which estimates the number of primes less than a given size. (In that case 
the number of primes less than $e^{L}$ is asymptotic to $e^{L} / L$ for large $L$.)

Mirzakhani looked at what happens to the "prime number theorem for geodesics" when one considers only the closed geodesics that are simple, meaning that they do not intersect themselves. The behavior is very different in this case: the growth of the number of geodesics of length at most $L$ is no longer exponential in $L$ but is of the order of $L^{6 g-6}$, where $g$ is the genus. Mirzakhani showed that in fact the number is asymptotic to $c \cdot L^{6 g-6}$ for large $L$ (going to infinity), where the constant $c$ depends on the hyperbolic structure.

While this is a statement about a single, though arbitrary, hyperbolic structure on a surface, Mirzakhani proved it by considering all such structures simultaneously. The complex structures on a surface of genus $g$ form a continuous, or non-discrete, space, since they have continuous deformations. While the underlying topological surface remains the same, its geometric shape changes during a deformation. Riemann knew that these deformations depend on $6 g-6$ parameters or "moduli", meaning that the "moduli space" of Riemann surfaces of genus $g$ has dimension $6 g-6$. However, this says nothing about the global structure of moduli space, which is extremely complicated and still very mysterious. Moduli space has a very intricate geometry of its own, and different ways of looking at Riemann surfaces lead to different insights into its geometry and structure. For example, thinking of Riemann surfaces as algebraic curves leads to the conclusion that moduli space itself is an algebraic object called an algebraic variety.

In Mirzakhani's proof of her counting result for simple closed geodesics, another structure on moduli space enters, a so-called symplectic structure, which, in particular, allows one to measure volumes (though not lengths). Generalizing earlier work of G. McShane, Mirzakhani establishes a link between the volume calculations on moduli space and the counting problem for simple closed geodesics on a single surface. She calculates certain volumes in moduli space and then deduces the counting result for simple closed geodesics from this calculation.

This point of view led Mirzakhani to new insights into other questions about moduli space. One consequence was a new and unexpected proof of a conjecture of Edward Witten (a 1990 Fields Medalist), one of the leading figures in string theory. Moduli space has many special loci inside it that correspond to Riemann surfaces with particular properties, and these loci can intersect. For suitably chosen loci, these intersections have physical interpretations. Based on physical intuition and calculations that were not entirely rigorous, Witten made a conjecture about these intersections that grabbed the attention of mathematicians. Maxim Kontsevich (a 1998 Fields Medalist) proved Witten's conjecture through a direct verification in 1992. Fifteen years later, Mirzakhani's work linked Witten's deep conjecture about moduli space to elementary counting problems of geodesics on individual surfaces.

In recent years, Mirzakhani has explored other aspects of the geometry of moduli space. As mentioned before, the moduli space of Riemann surfaces of genus $g$ is itself a geometric object of $6 g-6$ dimensions that has a complex, and, in fact, algebraic structure. In addition, moduli space has a metric whose geodesics are natural to study. Inspired by the work of Margulis, Mirzakhani and her co-workers have proved yet another analogue of the prime number theorem, in which they count closed geodesics in moduli space, rather than on a single surface. She has also studied certain dynamical systems (meaning systems that evolve with time) on moduli space, proving in particular that the system known as the "earthquake flow", which was introduced by William Thurston (a 1982 Fields Medalist), is chaotic.

Most recently, Mirzakhani, together with Alex Eskin and, in part, Amir Mohammadi, made a major breakthrough in understanding another dynamical system on moduli space that is related to the behavior of geodesics in moduli space. Non-closed geodesics in moduli space are very erratic and even pathological, and it is hard to obtain any understanding of their structure and how they change when perturbed slightly. However, Mirzakhani et al. have proved that complex geodesics and their closures in moduli space are in fact surprisingly regular, rather than irregular or fractal. It turns out that, while complex geodesics are transcendental objects defined in terms of analysis and differential geometry, their closures are algebraic objects defined in terms of polynomials and therefore have certain rigidity properties. This work has garnered accolades among researchers in the area, who are working to extend and build on the new result. One reason the work sparked so much excitement is that the theorem Mirzakhani and Eskin proved is analogous to a celebrated result of Marina Ratner from the 1990s. Ratner established rigidity for dynamical systems on homogeneous spaces-these are spaces in which the neighborhood of any point looks just the same as that of any other point. By contrast, moduli space is totally inhomogeneous: Every part of it looks totally different from every other part. It is astounding to find that the rigidity in homogeneous spaces has an echo in the inhomogeneous world of moduli space.

Because of its complexities and inhomogeneity, moduli space has often seemed impossible to work 
on directly. But not to Mirzakhani. She has a strong geometric intuition that allows her to grapple directly with the geometry of moduli space. Fluent in a remarkably diverse range of mathematical techniques and disparate mathematical cultures, she embodies a rare combination of superb technical ability, bold ambition, far-reaching vision, and deep curiosity. Moduli space is a world in which many new territories await discovery. Mirzakhani is sure to remain a leader as the explorations continue.

\section{References}

M. Mirzakhani, "Simple geodesics and Weil-Petersson volumes of moduli spaces of bordered Riemann surfaces", Inventiones Mathematicae, 2007.

M. Mirzakhani, "Weil-Petersson volumes and intersection theory on the moduli spaces of curves", Journal of the American Mathematical Society, 2007.

M. Mirzakhani, "Growth of the number of simple closed geodesics on hyperbolic surfaces", Annals of Mathematics, 2008.

M. Mirzakhani, "Ergodic theory of the earthquake flow", International Mathematics Research Notices, 2008.

A. Eskin and M. Mirzakhani, "Invariant and stationary measures for the $S L_{2}(R)$ action on moduli space", preprint 2013; see arXiv:1302.3320.

A. Eskin, M. Mirzakhani, and A. Mohammadi, "Isolation, equidistribution, and orbit closures for the $S L_{2}(R)$ action on moduli space”, preprint, 2013; see arXiv:1305.3015.

\section{Biography}

Born in 1977 in Tehran, Iran, Maryam Mirzakhani received her $\mathrm{PhD}$ in 2004 from Harvard University, where her advisor was Curtis McMullen. From 2004 to 2008 she was a Clay Mathematics Institute Research Fellow and an assistant professor at Princeton University. She is currently a professor at Stanford University. Her honors include the 2009 Blumenthal Award for the Advancement of Research in Pure Mathematics and the 2013 Satter Prize of the American Mathematical Society.

\section{Subhash Khot}

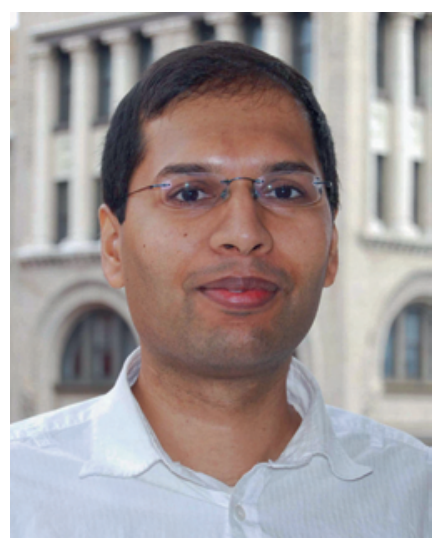

Typically, major math prizes are given for major results. But in this case, Subhash Khot is receiving the Nevanlinna Prize in large part for a conjecture-and even more surprisingly, one whose truth experts can't yet decide on.

But Khot's Unique Games Conjecture has already amply proven its value, even should it ultimately be disproven. It has cast a bright light on previously dim areas of computational complexity and provided critical insight-and, yes, Khot has also used it to prove major results, ones that stand regardless of its truth.

The conjecture has opened up a particularly fruitful way of addressing the central question of the field of computational complexity: How hard are problems to solve? More precisely, if you found the cleverest possible way to solve a particular problem, how quickly could a computer find the answer using it?

Computer scientists are nearly certain that some problems are so difficult that computers can't reliably find the answer at all, at least not in any reasonable amount of time (such as before the universe ends). That's the famous conjecture known as $\mathrm{P} \neq \mathrm{NP}$, and it has resisted proof for four decades-though computer scientists have only become more convinced that it must be true over time.

So many researchers have moved on to the next question: If a problem is too hard for a computer to solve quickly and precisely, can it at least find a good approximation? In the real world, after all, a good approximation is usually enough.

Before Khot's work, researchers had found a few problems for which the answer was no, but for most problems, they had little idea. Khot found a remarkably simple problem (called Unique Games) that seems to encapsulate what makes many problems hard to solve even approximately in a reasonable amount of time. His conjecture is that it's not just hard but impossible to reliably find an approximate answer to Unique Games reasonably quickly. In a certain precise, technical sense, Unique Games seems to be the simplest really, really hard problem.

Whether he's right or wrong, his problem has "cleaved nature at its joints," as the early taxonomist Carl Linnaeus put it. The conjecture is proving to be a kind of lever point, a spot where applying effort yields big results. Assuming the conjecture is true, Khot and others have shown that the vast majority of problems computer scientists care about also can't be approximated. Not only that, but the conjecture has shed light on seemingly unrelated problems in geometry, Fourier analysis, and even the mathematics of foams and voting, and those results don't rely on its truth.

The Unique Games problem is an elaboration of one that a six-year-old could play with. Imagine that you have a box of crayons and a drawing of a bunch of bubbles, some of which have lines connecting them. (Computer scientists call such drawings "networks.")

Can you find an efficient way to color in the bubbles so that any two connected ones are different colors? 
If your box has only two crayons (say yellow and purple), you can figure this out quite efficiently. Start with an arbitrary bubble and color it yellow. Since all the bubbles connected to it now have to be purple, color them in. Continue this way until you've either managed to color in the whole network or you've found a bubble that's connected to both yellow and purple bubbles, making the project impossible.

If you add just one more crayon, though, this method fails, because when you color the first bubble yellow, you don't know what color the connected ones have to be. So if you get to a bubble you can't color in without breaking the rules, you don't know if a different selection earlier would have solved the problem. The difficulty isn't just with that method-no other method will reliably and efficiently solve the problem either. It been proven to be NP-hard-in other words, effectively impossible.

Khot altered this problem slightly. He made it easier than the ordinary three-crayon problem by providing a rule so that whenever any bubble is colored in, the color of all connected bubbles is fixed. Then an algorithm like the earlier one applies, and it's easy enough to determine if the network can be colored in without breaking the rules. But for networks that are duds-ones that can't be colored in-Khot asked this: Which coloring breaks the fewest rules possible?

The Unique Games Conjecture (UGC) is that if you have lots of colors, you'll never find an efficient method to color in the drawing that's anywhere close to the best one no matter how clever you are.

Khot developed the conjecture in 2001 (in a slightly different formulation, which the name was derived from). A couple of years later, computer scientists got the first glimmer of its importance, when Khot and others found that if the UGC was true, then they could find firm limits on how well many other problems could be approximated.

Here's one: Imagine at a celebrity-studded party, guests sought out the stars to shake hands with them (but non-celebrity partygoers kept their hands to themselves with one another). If you know only who shook hands with whom, can you figure out the minimum number of celebrities that might have been at the party? A simple algorithm can find an approximate solution for this, but it might specify as much as twice as many celebrities as are really needed. Computer scientists long imagined you could do better with a more sophisticated algorithm, but in 2003, Khot, together with Oded Regev, showed that if the UGC is true, they were wrong. That simple algorithm is the best you can do.

Khot's next success came in 2005. Together with Ryan O’Donnell, Elchanan Mossel and Guy Kindler, Khot found that the UGC implied a similar limit for approximating solutions to a problem called "Max Cut," which asks you to take a network and split it into two groups so that the maximum number of edges passes between them. Then in 2008, Prasad Raghavendra showed that if the UGC is true, a very simple method can find the best approximations for an enormous class of problems called "constraint satisfaction problems." With this, computer scientists know exactly how well nearly any problem can be approximated.

Of course, the caveat is that all these results depend on the UGC being true. If it turns out to be false, the entire sparkling, beautiful theory is a mirage.

But the conjecture has proved remarkably powerful independent of its truth. In the process of using the UGC to discover how well other problems could be approximated, Khot and others have proven several significant theorems in other areas, including geometry and Fourier analysis.

These implications have even ranged as far as voting theory. Once all the votes in an election are cast, there are a variety of ways of determining the winner. One of the most obvious is that the election goes to the candidate with the majority of votes, but there are other choices too, such as the American electoral college system. Khot and his co-authors used intuition from the UGC to propose that majority-rules is the method for counting votes in which a few miscounted votes is least likely to change the election result. This was indeed confirmed later by others.

Another group was working on proving that the UGC was true, and while their method failed, the work led them to another discovery: They found a shape that in a certain sense lies halfway between a square and a circle (though in many more than two dimensions). Like a square, copies can be placed next to each other horizontally and vertically to fill a whole space without gaps or overlaps, forming a multidimensional foam. But its perimeter is much smaller than a square-it's closer to that of a circle, the object which has the smallest perimeter for the area contained.

In the meantime, other researchers have worked to prove that the UGC is wrong, with equal lack of direct success, but with equal collateral benefits. Although they haven't yet succeeded in finding an algorithm that can efficiently find a good approximate solution to Unique Games, they have developed some excellent new algorithmic methods for other circumstances.

Efforts to prove the conjecture, to disprove it, and to discover its consequences have all proven enormously fruitful. The Unique Games Conjecture will be driving research in theoretical computer science for many years to come. 


\section{Stanley Osher}

Stanley Osher is a one-man bridge between advanced mathematics and practical, real-world problems. Time and time again, he has engaged deeply with the world of the engineers and applied scientists and then developed mathematical techniques to solve their problems with unprecedented power, speed and elegance. And because he so finely tunes his solutions to their needs, his techniques have been widely adopted and have had an extraordinarily broad impact, helping to catch criminals, create animated movies, improve MRI scans, design computer chips, and much more.

One of his major contributions was developing the method of level sets with James Sethian. The basic problem is to mathematically describe a changing shape. Imagine, for example, that a drop of oil is floating in water, and you want to write an equation that will predict how its shape will change with currents in the water. A traditional way to do this is to picture a set of buoys along the edge of the drop, connected by stretchy ropes. Moment by moment, you can then describe how the buoys move, giving you a good approximation of the overall shape.

That method doesn't work so well, though, if your oil drop splits in half, as they sometimes do. It's not so obvious how to describe that with the buoys, since you now also need to cut the ropes between them and retie them. Or, suppose that two different oil drops merge-then some of your buoys disappear completely, or fall inside the other oil drop, or something. It gets messy.

Osher and Sethian suggested an entirely new approach. They imagine that the outline of the oil drop is a clean horizontal slice of some three-dimensional object. The rest of the shape of the object doesn't much matter, as long as the slice is the right shape. They then apply the physical laws affecting the drop of oil (for the currents in the water, for example) to the entire three-dimensional object. To find the shape of the oil drop at another moment, they simply take the same horizontal slice of the object later. If the oil drop splits, the three-dimensional object has developed two separate humps. And if two drops come together, two "legs" of the object have converged.

Although turning the problem of predicting the evolution of a two-dimensional object into predicting that of a three-dimensional one seems like it would only make things more complicated, it solves all the bookkeeping problems with the buoy-and-ropes method and gives a simple, powerful, clean representation that can handle any odd thing the oil drop might do.

This relatively simple idea turns out to be extremely powerful. For example, the level set method
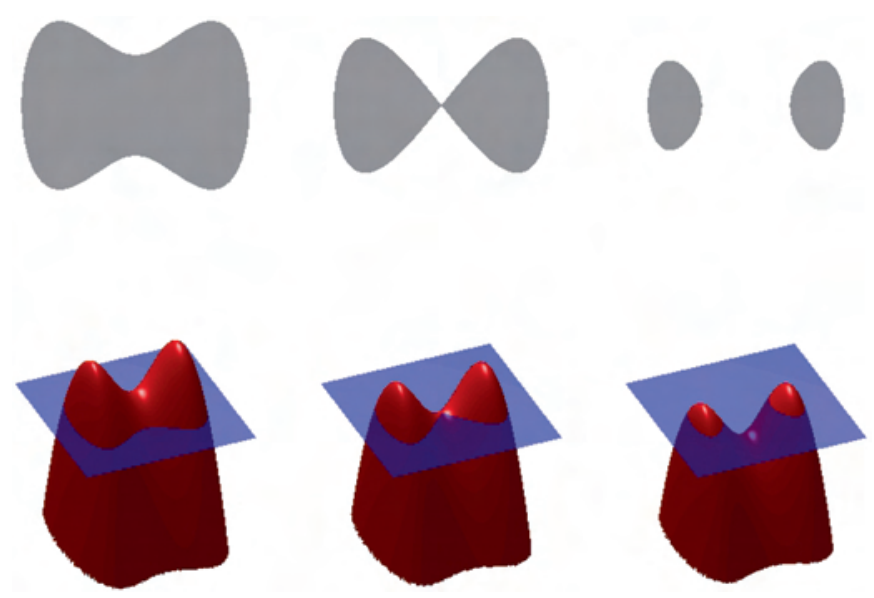

Figure 1. Horizontal slices of the three-dimensional red object on the bottom show the shape of an oil drop as it changes over time. This representation can gracefully handle behavior like the drop cleaving in two. Credit: Wikimedia.

is now being used by every single major film animation company to animate fluids, including Pixar, Disney, ILM, Dreamworks, and more. It allows animators to apply the true laws of physics to the fluid, creating far more realistic images. The giant whirlpool maelstrom in Pirates of the Caribbean 3 and the dragon's flaming breath in Harry Potter and the Goblet of Fire, for example, were created using level sets. One of Osher's students, Ron Fedkiw of Stanford University, won an Academy Award for his work on film animation using these methods.

More practically, level set methods are useful for predicting weather, designing computer chips, identifying the source of an earthquake, modeling the growth of tumors, analyzing medical scans, and much, much more.

Another area that Osher has revolutionized is modeling the way that supersonic jets slice through the air. Air flows smoothly around planes flying at ordinary speeds, but when jets approach the speed of sound, the air can't get out of the way fast enough. As a result, the density, pressure, temperature and velocity of the air change essentially instantaneously. In mathematical terms, this instantaneous change is called a "discontinuity," and it's a really big problem, because traditional methods all depend on incremental changes. Osher, together with Amiram Harten, Bjorn Engquist and Chi-Wang Shu, developed new mathematical techniques that can handle these discontinuities. This enables computer modeling of the design of new supersonic jets.

Osher then applied similar ideas to an entirely different problem: sharpening blurry images. The line in a photograph between the dark leg of a table and 

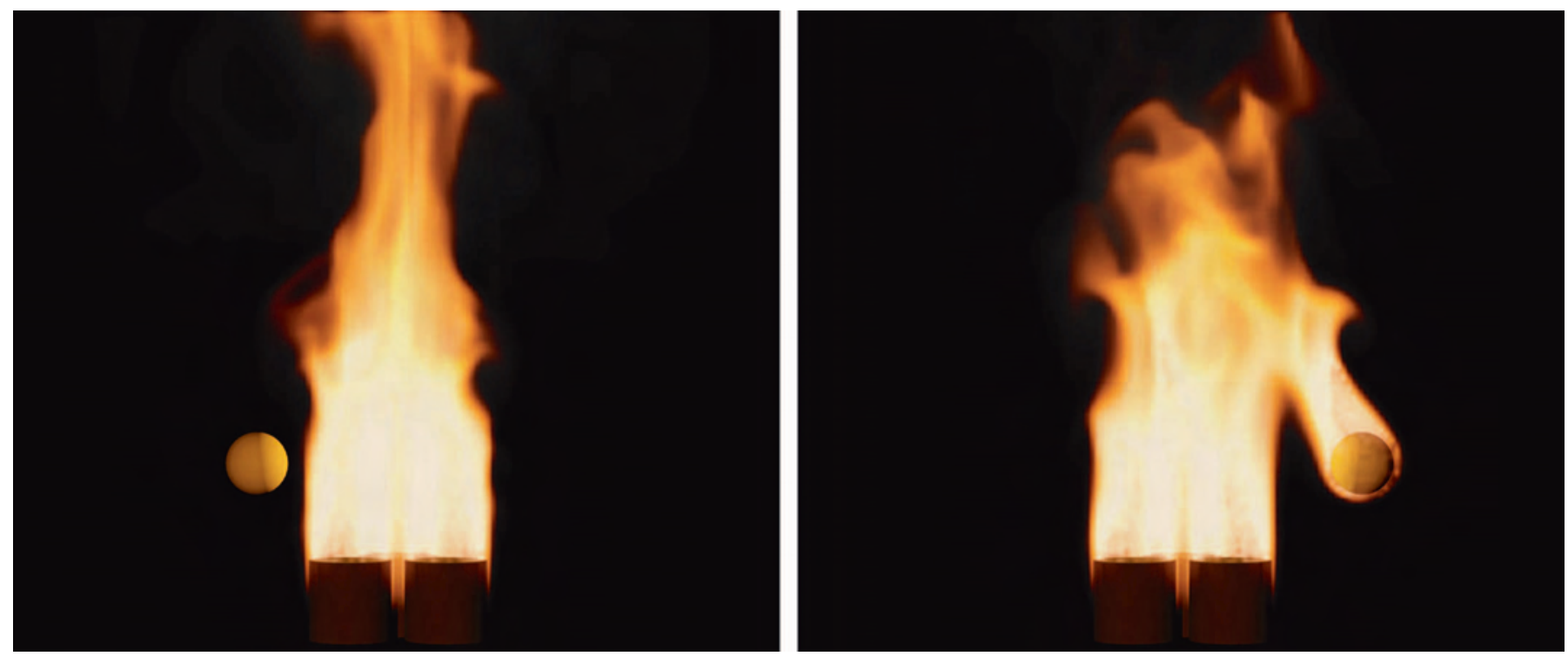

Figure 2. This is not a photograph. This is a computer simulation of a ball flying through a flame and catching fire. It was created using Osher's level set methods. Credit: Ron Fedkiw, Duc Nguyen and Doug Enright.

a light background is another example of a discontinuity. Together with Leonid Rudin, Osher applied the mathematical techniques he had developed for modeling supersonic jets to images and was able to pull out and sharpen these discontinuities.

Their techniques also came from the recognition that much of the fuzz in a blurry image isn't random: It comes from physical processes like the shake of the hand holding the camera. Identifying these processes and then reversing them reduces the blur. That's easier said than done, though, because information is lost when the image is blurred. Osher's methods can recover some of that by combining information from multiple images. And he developed very efficient algorithms to carry out the transformation.

Never content with merely developing theoretical methods, Osher (together with Rudin) created a company, Cognitech, to commercialize them. The most famous success of the company came during a trial after the 1992 Los Angeles riots. Rioters attacked a truck that happened to be driving through the area, throwing rocks at it, dragging the driver out of the cab, beating him to unconsciousness, and breaking his skull in 91 places. The entire attack was filmed by a TV helicopter (one of the attackers even danced over the victim's unconscious body and flashed gang symbols to the helicopter). The footage was blurry, though, so prosecutors turned to Cognitech for help identifying the attackers. Investigators focused on a speck on the arm of one of the men-less than $1 / 6,000$ th the size of the total photograph-and the algorithms revealed it to be a rose-shaped tattoo. The man was later identified as Damian Monroe Williams, and he was convicted of the attack. Cognitech continues to be used by police departments across the country.

Another of Osher's clever algorithms allows people to get better MRI scans faster. Building on the compressive sensing ideas of David Donoho, Emmanuel Candes and Terence Tao, he generalized his image processing methods to any situation in which you want to present information using as little data as possible. Jpeg, for example, is an algorithm that stores images using fewer bytes, while losing only a small amount of detail. He developed algorithms to do the same thing in reverse, so that the scan gathers its data in compressed format, requiring less of it to get a clear view. Another example where these techniques apply is the "Netflix problem," predicting what movies you will like from your favorites. The movies you've seen and liked are the data represented in compressed form, and the problem is to uncompress that to discover all the movies you will like.

As diverse as Osher's impacts on the world have been, they've all come from clever, efficient algorithms drawing on deep mathematics. "I write the algorithms that make the computer sing," Osher told the Los Angeles Times. "I'm the Barry Manilow of mathematics."

\section{Phillip Griffiths}

Phillip Griffiths is a singular figure in mathematics. For more than 50 years, he has been a leader in research, making contributions at the highest level in 

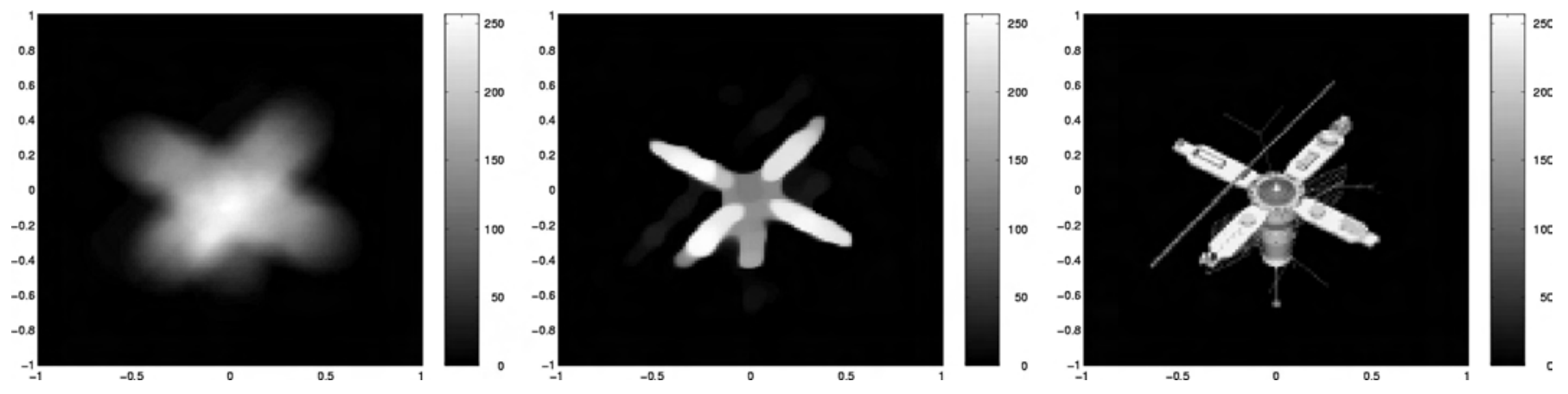

Figure 3. The leftmost image is a blurry picture of TK. Osher's methods sharpen it into the middle image. The rightmost image is a picture of the same thing that is in focus.

several areas, most notably algebraic geometry and differential geometry. He has also been an outstanding teacher and mentor for young people entering mathematics. His exposition has garnered accolades, and his books have had a lasting influence. On top of these contributions to the field of mathematics, Griffiths has had a substantial impact on the entire scientific enterprise the world over, through his extensive work on science policy, through his leadership of the Institute for Advanced Study for more than a decade, and through his work on behalf of science in the developing world. To have contributed at such a high level to so many different aspects of mathematical and scientific life is truly extraordinary.

Right after he received his $\mathrm{PhD}$ from Princeton University in 1962, under the direction of Donald Spencer, Griffiths went to the University of California, Berkeley, where he was a Miller Fellow. There, he came into contact with Shiing-Shen Chern (1911-2004), after whom the Chern Medal is named. One of the towering figures of 20th century mathematics, Chern was a geometer of wide interests and deep insights. He also had a profound sense of responsibility for developing the culture and community of mathematics. He made a lasting impression on the young Griffiths. The two became collaborators and lifelong friends.

In his mathematics research, Griffiths is known not so much for having proved big theorems or for things named after him-though he certainly has both. Rather, he is known more for pioneering new approaches or strategies that have proven very fruitful, for developing connections between areas that had previously seemed unrelated, and for opening new lines of research that he and others then pursued. He also has the ability, despite the formidable technical machinery used in his work, to hold fast to the intuitive heart of the problem at hand, noted Robert Bryant of Duke University, who served as chair of the 2014 Chern Medal selection committee. "Even when mathematicians discuss very abstract geometric concepts, they often speak as though there are tangible objects being represented and attach an almost physical sense to them," said Bryant. "Exactly how this sort of metaphorical sense contributes to our understanding of the concepts is mysterious, but it is frequently the hallmark of great insight. Griffiths has an amazingly strong ability to invoke those kinds of intuitions and to communicate them to others."

One of Griffiths' great contributions has been to perceive this intuition in the work of past mathematical masters and reinterpret it in modern terms. For example, mathematicians looked askance at the work of the Italian school of algebraic geometry of the late 19th and early 20th century because the work did not conform to new standards of rigor that arose afterward. The Italian algebraic geometers nevertheless had a genius for geometric intuition. Griffiths imbibed this intuition and made it precise, using modern techniques. He also absorbed and revived interest in the work of Élie Cartan (1869-1951) on exterior differential systems. While it was full of tremendous insights, Cartan's work had been neglected because was difficult to read and had not been put into a systematic framework. After Griffiths and his collaborators developed and expanded on ideas from Cartan's work, exterior differential systems went on to have a significant impact on the theory of partial differential equations. "There was a kernel of beautiful geometric ideas that ran through these classic works and-once you got through the old-fashioned language and notations - an extraordinary relevance to modern problems," said Mark Green of the University of California at Los Angeles, who will be presenting the laudatio for Griffiths. "Griffiths was a great believer in the power of deep geometric ideas, and he encouraged his students to engage with these classic books and papers."

Four volumes of the selected works of Griffiths have appeared: Analytic geometry, algebraic geometry, variations of Hodge structures, and differential systems. There is also ample material for a fifth volume, which is under consideration. This staggering output ranges over an enormous variety of topics. 
Nevertheless, one can still perceive some unifying and powerful geometric ideas that run through his work. An example can serve to illustrate the nature of these ideas. A fundamental distinction in mathematics is between algebraic functions, such as the square root of $x$, and transcendental functions, such as sine and cosine, which cannot be expressed algebraically. An important insight that emerged in algebraic geometry over the course of the 19th century is that objects described in terms of algebraic equations can be productively studied using transcendental functions; this gives rise to the subject known as transcendental algebraic geometry. For algebraic curves in the plane, one aspect of these transcendental methods was embodied in a construction known as the period map. However, when one goes beyond curves to higher dimensions, genuinely new phenomena occur that no one had anticipated until the work of Griffiths. He found an innovative way to bring together modern methods, such as deformation theory and Hodge theory, with the classic framework.

Not far off from these developments lies one of the major challenges in mathematics, the Hodge Conjecture, which has been designated as one of the million-dollar Millennium Prize Problems of the Clay Mathematics Institute. W. D. V. Hodge (1903-1975) noted that to certain objects in algebraic geometry-namely, algebraic cycles-one can associate a particular type of object in topology, a "Hodge class". The Hodge Conjecture asks whether one can reverse this association: Can one take a Hodge class and find an algebraic cycle with rational coefficients to which it is associated? While no one has yet proved the Hodge Conjecture, Griffiths' work has been a potent force in shedding light on the contours of that problem and shaping much of the research that has been done on it.

Often mathematicians are categorized as "problem solvers" or "theory builders"; Griffiths does not quite fit into either group. "He's an 'understander'," said Bryant. "He wants to understand where an idea can go, its connections to other ideas, and how it might illuminate a problem he's considering." This drive to understand has made Griffiths an excellent communicator, not only among fellow researchers but for students as well. He has had $29 \mathrm{PhD}$ students, many of whom have gone on to outstanding careers themselves; altogether, he has about 460 doctoral "descendents". Griffiths' mathematical writing is known for its clarity and polish as well as for the way it opens new directions for research. A prominent example is his paper "Periods of integrals on algebraic manifolds", which appeared in the Bulletin of the American Mathematical Society in 1970 and received the AMS Steele Prize the following year. His books have also had a wide influence, particularly
Principles of Algebraic Geometry, written with his former PhD student Joseph Harris. Universally known by the shorthand "Griffiths and Harris", this textbook has become a standard reference for generations of students.

After his Miller Fellowship at UC Berkeley, Griffiths became a faculty member there. In 1967, he moved to Princeton University and in 1972 to Harvard. His appointment in 1983 as provost of Duke University began his work in administration, which, surprisingly, did not slow down at all his research output. In 1991, he became director of the Institute for Advanced Study (IAS) in Princeton, one of the world's foremost research centers, particularly in the areas of mathematics and theoretical physics. Under his 12-year directorship, the IAS launched several new initiatives, including programs in theoretical computer science and theoretical biology. Also during this time, three new buildings were completed, among them Simonyi Hall, which now houses the IAS School of Mathematics.

Griffiths has been much sought after for his wise council in matters of science and educational policy. As Green put it, "He is a person everyone trusts to be fair and judicious." Among the most prominent roles Griffiths played was as chair of COSEPUP, the Committee on Science, Engineering, and Public Policy of the National Academies, from 1992 until 1999. In that time, COSEPUP issued two especially influential reports, one on reshaping graduate education in science and engineering, and the other on the various facets of being an effective mentor in these areas. Griffiths also served on the National Science Board, the policymaking body of the National Science Foundation (1991-1996) and as secretary of the International Mathematical Union (1999-2006).

While director of the IAS, Griffiths got to know James Wolfensohn, president of the World Bank, who at the time served as an IAS trustee. Inspired by this contact, Griffiths launched the Science Initiative Group (SIG), an international team of leading scientists that aims to help developing countries build scientific capacity. Rather than imposing goals and frameworks from outside, SIG helps native-born scientists to identify critical national needs and to build the educational and research infrastructure required to address them. SIG's first effort was the Millennium Science Initiative, a research and education program that was funded mainly by the World Bank and that reached developing countries in Africa, Asia, and Latin America. Building on this experience, SIG launched RISE (Regional Initiative in Science and Education), which is funded by the Carnegie Corporation of New York and managed jointly by SIG and the African Academy of Sciences. RISE supports university-based research and training networks in 
sub-Saharan Africa, with the goal of preparing $\mathrm{PhD}$ level scientists and engineers. RISE has put special emphasis on participation by African women, who have long been underrepresented in the sciences.

At 76 years of age, Griffiths shows little sign of slowing down. Now a professor emeritus at the IAS, he remains deeply involved with SIG. Over the past year he has teamed with other U.S. leaders in mathematics and science on a program that aims to effect constructive change in post-secondary mathematics education. And he is still doing research: His most recent paper, written with Mark Green, was posted on the arXiv preprint server in May 2014. With his distinguished legacy in mathematics research, his profound impact on young people in the field, and his contributions to supporting research and education around the globe, Griffiths is an inspiration to many-but not one who is easily emulated, Green noted. To take on as many projects as Griffiths has, "the rest of us would need extra hours in the day."

\section{Adrián Paenza}

Adrián Paenza has been awarded with the Leelavati Prize 2014 because of his decisive contribution in changing the mind of a whole country about the way that mathematics is perceived in daily life. He has accomplished this through his books, his TV programs, and his unique gift of enthusiasm and passion in communicating the beauty and joy of mathematics.

At a very young age he received his $\mathrm{PhD}$ degree in Mathematics and taught at the University of Buenos Aires between 1979 and 2002. During many years he pursued a parallel life as a successful TV Sports Journalist, and also as a Political Journalist. Since 2003 he found a way to integrate his mathematical background with his journalism experience: he started a shining career as a Science Journalist.

He has been the host of the long running weekly TV program "Científicos Industria Argentina" ("Scientists Made in Argentina"), currently in its twelfth consecutive season in an open TV channel. Each program, with a beautiful and attractive interface, consists of interviews to mathematicians and scientists of very different disciplines, and ends with a mathematical problem, whose solution is given in the next program.

He also has been the host of the TV program "Alterados por Pi” (“Altered by Pi”), a weekly half hour show exclusively dedicated to popularize maths. The show is recorded in front of a live audience in several public schools around the country.

Since 2005, he has written a weekly column about general science, but mainly about mathematics, on the back page of "Página 12", one of Argentina's three national newspapers. His articles include historical notes, teasers and even proofs of theorems.

He wrote eight books dedicated to the popularization of mathematics: five under the name "Matemática... ¿estás ahí?" ("Math... are you there?), published by Siglo XXI Editores, which have sold over a million copies. The first of the series, published in September 2005, headed the lists of best sellers for a record of 73 consecutive weeks, and is now in its 22nd edition. The enormous impact and influence of these books has extended throughout Latin America and Spain; they have been published in Portugal, Italy, Czech Republic, Germany, and recently translated also to Chinese language for a next coming edition.

It is remarkable that, by his personal generosity, all his books were made freely available at the Department of Math of the University of Buenos Aires from the first day of publication and sale.

Paenza also has been always a champion of the public and free education. He introduced the idea (which was later implemented by the Argentinean Education Minister) of developing a Federal program for digital literacy, inspired by the program "One laptop per child". His books are distributed with no cost in these free laptops.

Nicholas Negroponte has said about his nomination:

"I know Adrián and his work very well. He is an inspiration and a hero for mathematics. His presence in the USA and Argentina has inspired many young people...not to mention the whole Spanish speaking world. I cannot imagine a more qualified nomination."

\section{And John Sulston said:}

\footnotetext{
"How delighted I am that Adrián Paenza is nominated for the prize! It is clear from the record that he has an enormous range and reach, and is widely appreciated for his lucid presentations. He was intensely interested not only in the science, but also by the insistence of me and my colleagues on open data release. This is a theme that he has returned to himself many times, defending the importance of public and free education for everyone without cost restrictions. He and I therefore found a shared passion during our conversation, and I greatly admire the work he has done. I think Adrián is a wonderful candidate for the Leelavati Prize."
} 